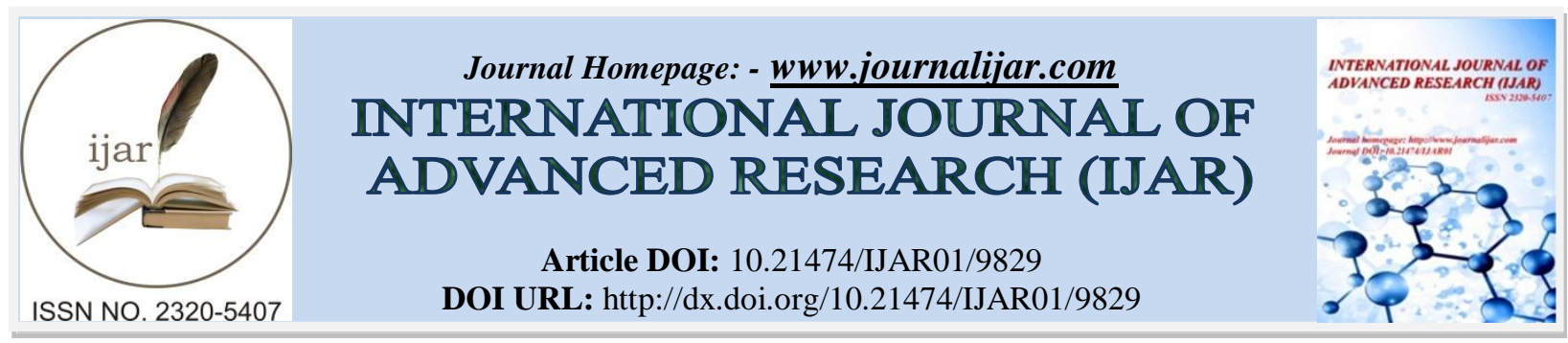

RESEARCH ARTICLE

\title{
ROLE OF MDCT IN DIAGNOSIS AND RADIOLOGICAL CHARACTERIZATION OF NEUROBLASTOMA.
}

\section{Lina Mohsin Rida.}

\section{Manuscript Info}

\section{Manuscript History}

Received: 08 August 2019

Final Accepted: 10 September 2019

Published: October 2019

Key words: MDCT, neuroblastoma, metastasis.
Abstract

Background: Neuroblastoma is one of the most common solid malignant neoplasms in childhood. Accounts for $15 \%$ of cancer deaths, mostly in the first two years. It has an infiltrative growth pattern, with early metastatic disease. Diagnosis of the tumor is important prior to treatment .

Aim of The Study: To evaluate the accuracy of MDCT in diagnosis and radiological characterization of neuroblastoma and to assess its extent , common and uncommon locations.

Material and Methods: Retrospective study was carried out in radiology department at Karbala teaching hospital for pediatrics between January 2016 and November 2018, on twenty four patients, $8(33.3 \%)$ were males and $16(66.7 \%)$ were females, mean age $(3.55$ \pm 2.21 years), who had a radiological impression of and reported as neuroblastoma on MDCT examination and patients those who had a final diagnosis of neuroblastoma as confirmed by histopathological examination of the surgical specimens. All patients had undergone native and contrast enhanced CTS to the area of interest.

Results: The diagnostic accuracy of MDCT in diagnosis of neuroblastoma was (83.3\%) and sensitivity value was (90.9\%). Most common location was in the abdomen (77.3\%) and about (13.64\%) at the superior and posterior mediastinum. The least common sites were the neck and pelvis and the percentage was (4.5\%) for each. Most of the tumor masses are large at presentation, extend beyond the midline, with diagrammatic extension and the presence of distant metastasis, $18(81.8 \%)$ of patients had tumors of stages 3 or 4 .

Conclusion: MDCT is a valuable tool in diagnosis and radiological characterization of neuroblastoma.

Copy Right, IJAR, 2019,. All rights reserved.

\section{Introduction:-}

Neuroblastoma (NB) is the most common extracranial solid tumor of early childhood (8-10\%), the 4th after leukemia, lymphoma and brain malignancies ${ }^{(1)}$. Neuroblastoma arises from primitive neuroblasts of the embryonic neural crest, and therefore can occur anywhere within the sympathetic chain ${ }^{(2,3)}$,(figure 1).

The most common site of the primary tumor occurs within the abdomen (65\%). About half of these tumors arise from the adrenal medulla. Other common sites of neuroblastoma include the neck, chest and pelvis ${ }^{(4)}$. 
The diagnosis is based on histopathological examination of tumor tissue, supplemented with immunohistology or electron microscopy, and a finding of increased excretion of catecholamine metabolites in the urine ${ }^{(5)}$. The tumor is sometimes diagnosed from the presence of tumor cells in bone marrow aspirate. Staging is performed on the basis of clinical, radiological and surgical evaluation with histopathological correlation ${ }^{(5)}$. The International Neuroblastoma Staging System (INSS) was launched and was revised in $1993^{(6)}$. The different stages were defined as in (table 1).

Most children with neuroblastoma present between 1 and 5 years of age, median age 2 years, with a palpable abdominal mass. This may be an incidental finding in an otherwise healthy child or in a child clearly unwell from metastatic spread of the tumor. Abdominal distension, generalized skeletal pain or even arthritis type complaints, effects of hormone production and nonspecific findings from bone marrow involvement, such as weight loss, malaise, anemia, fever and irritability can be encountered ${ }^{(1)}$. In half of the patients with intra-spinal tumor extension, peripheral neurologic deficits and neurological symptoms from compression of the nerve roots or the cord may be present ${ }^{(1)}$. In less than $2 \%$ of cases, neuroblastomas can present with paraneoplastic syndromes: opsoclonusmyoclonus-ataxia syndrome or watery diarrhea ${ }^{(7,8)}$. It can be discovered incidentally during scanning for other reasons, e.g. chest radiograph for pneumonia in case of thoracic neuroblastoma ${ }^{(7,8)}$.

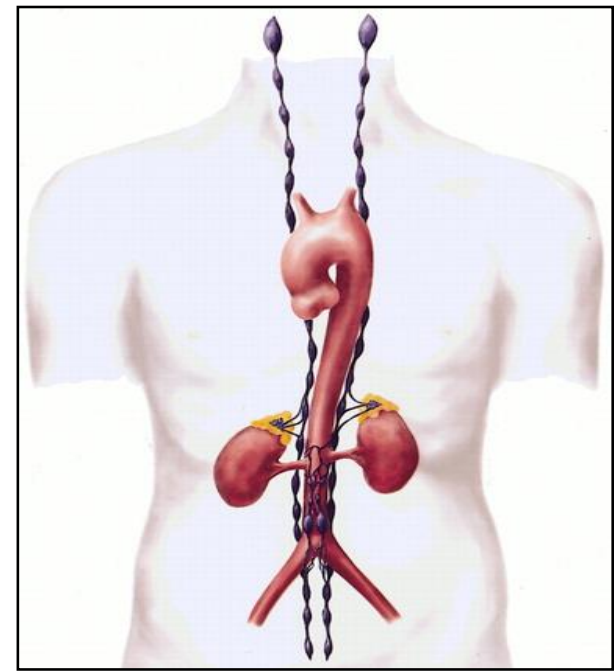

Figure 1:-Anatomic distribution of sympathetic ganglia. Diagram illustrates the sympathetic ganglia extending from the neck to the pelvis, including the adrenal medulla (sympathetic tissue shown in blue) ${ }^{(3)}$.

Table 1:-staging of neuroblastoma ${ }^{(5)}$

\begin{tabular}{lll}
\hline Stage & INSS & Imaging \\
\hline 1 & Localised tumour & Localised tumour \\
& Complete gross excision & Well-defined fat plane surrounds the tumour \\
$2 \mathrm{~A}$ & Localised tumour & Localised tumour \\
& Incomplete gross excision & Incomplete fat plane separation \\
2B & Localised tumour & Localised tumour \\
& Incomplete gross excision & Incomplete fat plane separation \\
& Ipsilateral lymph nodes positive & Ipsilateral lymph nodes $>1 \mathrm{~cm}$ \\
3 & Unresectable midline tumour with bilateral lymph nodes positive or & Growth beyond midline \\
& Unilateral tumour with contralateral lymph nodes positive & Encased vessels \\
& & Mesenteric or diaphragm involvement \\
& & Lymph nodes $>1 \mathrm{~cm}$ \\
& & Ascites \\
4 & Primary tumour with involvement of: remote lymph nodes, other organs & Primary tumour with involvement of: spinal canal, other organs \\
4S & $<1$ year & $<1$ year \\
& Localised tumour stage 1-2 & Localised tumour stage $1-2$ \\
& Metastases to organs but not bone & Metastases to organs but not bone \\
\hline
\end{tabular}




\section{Materials and methods:- \\ Patient selection and study design :}

A retrospective study was carried out in radiology department at Karbala teaching hospital for pediatrics between January 2016 and November 2018, on twenty four patients ( 8 boys and 16 girls).

\section{Inclusion criteria:}

Patients who had a radiological impression of and reported as neuroblastoma on MDCT examination and patients those who had a final diagnosis of neuroblastoma as confirmed by histopathological examination of the surgical specimens.

\section{Methods:}

All patients had undergone native and contrast enhanced CTS to the area of interest (abdomen, pelvic and chest), using Siemens, Somatom, 64 multi-slice CT scanner. The images were reviewed blindly by two specialist radiologists.

The tumors were assessed with regard to the location, size, homogeneity pre and post IV contrast administration , presence of calcification, necrosis, local and beyond the midline extension, kidney displacement, invasive growth, vascular involvement, intra-spinal, paravertebral, or mediastinal extension and distant metastases. Distinguishing between the primary tumor and the adjacent nodal disease is some times difficult and even said to be often impossible by G. Papaioannou et $\mathrm{al}^{(1)}$. Thus lymphadenopathy when suspected, it was considered to be present if lymph nodes more than $0.5 \mathrm{~cm}, 0.8 \mathrm{~cm}$ and $1 \mathrm{~cm}$ for mesenteric, para-aortic and cervical nodes respectively , in shortest axis and were separated from the primary tumor by a fat plane. The tumor was considered to be localized to site of origin when preserved fat plane surrounding the tumor, separating it from adjacent organs or tissues, this is seen on CT as a low-attenuating line, and was supposed to be a stage 1 tumor, absence of such a separation was considered a sign of possible tumor invasion ${ }^{(5)}$. If the tumor could not be demarcated from the surrounding tissues or organs as psoas muscle or the kidney, it was supposed to be a stage 2 tumor. A midline position of the tumor, growth beyond the lateral aspect of the opposite side of the spine and/or vascular encasement were considered as stage 3 . Vascular involvement was considered to be present if the tumor was encasing 180 degrees of the vessel or more of its circumference. Suspected diaphragmatic involvement and ascites together indicated a possible stage 3, but were not by themselves indicative of a stage 3 disease $^{(5)}$. Lung metastases, uni- or bilateral para-vertebral intrathoracic extension, intra-spinal extension, or liver, brain, or bone involvement indicated a stage 4 tumor. Bone involvement was considered positive when there was a lytic or sclerotic lesion. Pleural fluid may indicate a stage 4 tumor, but not without the presence of malignant $\operatorname{cells}^{(5)}$. Different stages are illustrated in (table 1).

\section{Results:-}

A total of 24 patients were included in the study, 8(33.3\%) were males and $16(66.7 \%)$ were females, their ages ranged from ( 5 months - to 8 years), with a mean age of ( $3.55 \pm 2.21$ years), table( 2 ). All had undergone native and contrast enhanced CTS. Twenty two patients were reported as neuroblastoma, twenty patients( $83.3 \%$ ) were accurately diagnosed as neuroblastoma as confirmed by histopathological study of the surgical specimen while there were two reported as Wilms tumor revealed to be a neuroblastoma(NB) as shown by table (3), making a total number of 22 patients having NB , 7 (31.8\%) males and 15(68.2\%) females(figure 2).

Total accuracy of MDCT is diagnosis of neuroblastoma was (83.3\%) and total sensitivity was ( $90.9 \%$ ).

Clinically more than two thirds presented with abdominal symptoms as mass, pain, and distension, others with diarrhea ( paraneoplastic syndrome), neck mass, one presented with Horner's syndrome ( had an upper mediastinal mass extending to neck root, figure 3), two presented with Hutchinson syndrome (presented with skeletal pain and palpable lumps due to bony metastases figure 4), one with Blueberry muffin syndrome( multiple cutaneous lesions figure 5) and one with Pepper syndrome( hepatomegaly due to extensive liver metastasis, figure 6).

First radiological detection was by abdominal ultrasounds and plain chest radiograph.

Most common location was in the abdomen (77.3\% ), more than half are found at the adrenal gland (figure 7), the remainings were found at the retroperitoneum(figure 8), namely the paravertebral sympathetic chain, around the celiac axis and organ of Zuckerkandl ( figure 9 ). About (13.64\%) at the superior and posterior mediastinum. The least common sites were the neck and pelvis and the percentage was (4.5\%) for each as shown by table (4). 
NB masses showed considerable extension, whether local on the same side in(50\%), crossing the midline (63.3\%); $(41 \%)$ for each of the above or below the diaphragm, para-vertebral and mediastinal extension, spinal extension (18.2\%), (figure 8 and 10) and the distant metastasis was (59.1\%), mostly to the bone (18.2\%). Renal displacement was found in $(70.5 \%$ regrading only the abdominal cases ), while invasive growth was found in $(22.7 \%)$, one case found to invade pancreatic body(figure 9). The suspected lymphadenopathy was seen in (63.3\%) and vascular encasement represented (81.8\%) of the cases, no vascular invasion. 14(63.63\%) of patient was considered as stage 4 , while $4(18.18 \%)$ of them were stage 3, 2 ( $9.09 \%)$ pateints were stage 2 and $2(9.09 \%)$ patients were stage $4 \mathrm{~S}$.

Most of the tumor masses $(95.5 \%)$ showed heterogeneity pre and post IV contrast administration (figure 10) and $(86.4 \%)$ showed necrosis. Calcification was seen in $(72.7 \%)$, figures (4,6,8 and 10).

Table 2:-Baseline characteristics of the studied sample.

\begin{tabular}{|l|l|l|l|l|}
\hline \multicolumn{2}{|l|}{} & Mean & Std. Deviation & Range \\
\hline \multicolumn{2}{|l|}{ Age (years) } & 3.55 & 2.21 & $05 / 12-8 y$ \\
\hline \multirow{2}{*}{ Gender } & Male & $8(33.3 \%)$ & & \\
\cline { 2 - 5 } & Female & $16(66.7 \%)$ & & \\
\hline Duration (months) & 30.39 & 0.871 & $1-23$ \\
\hline
\end{tabular}

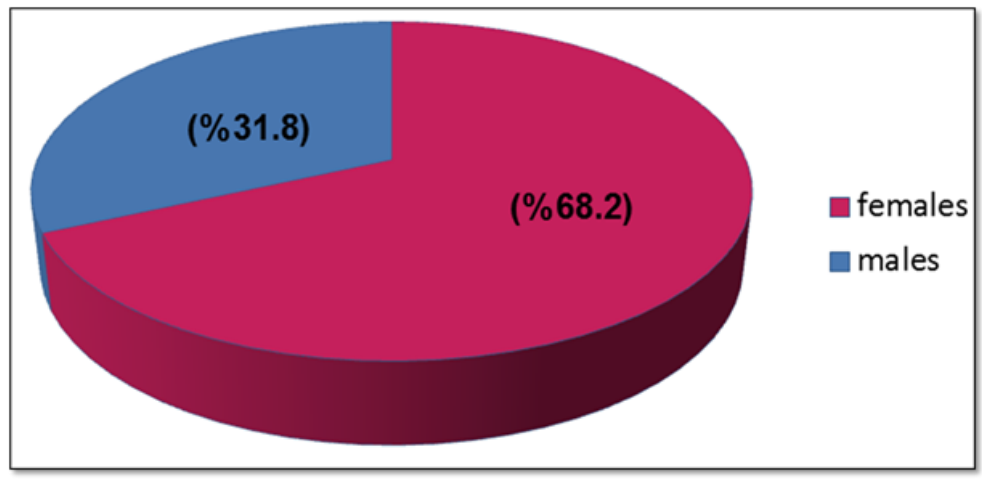

Figure 2:-Distribution of patients of neuroblastoma according to gender

Table 3:-Showing correlation of the CTS reports with histopathological result of the studies sample

\begin{tabular}{|l|l|l|l|l|}
\hline $\begin{array}{l}\text { No. of patients } \\
\text { included in the study. }\end{array}$ & \multicolumn{2}{l|}{ CTS report } & \multicolumn{2}{l|}{ Histopathological result } \\
\hline 24 & 22 & NB & 20 & NB \\
\cline { 3 - 5 } & & 1 & Adrenocortical ca \\
\cline { 3 - 4 } & & 1 & Extra renal Wilms T \\
\cline { 2 - 4 } & 2 & Wilms T & 2 & NB \\
\hline
\end{tabular}


Table 4:-Radiological CTS Finding of 22 patients truly diagnosed as NB

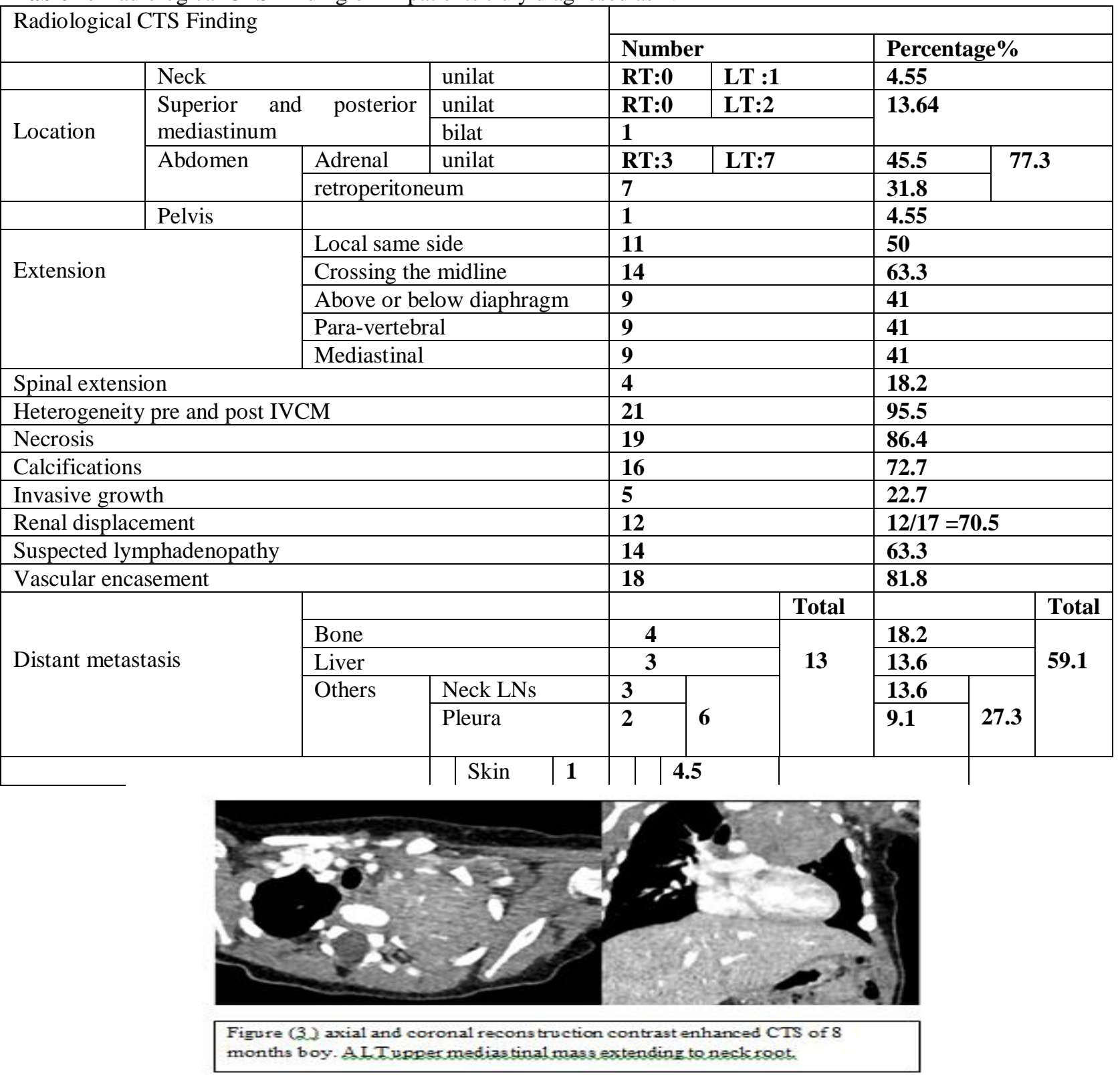

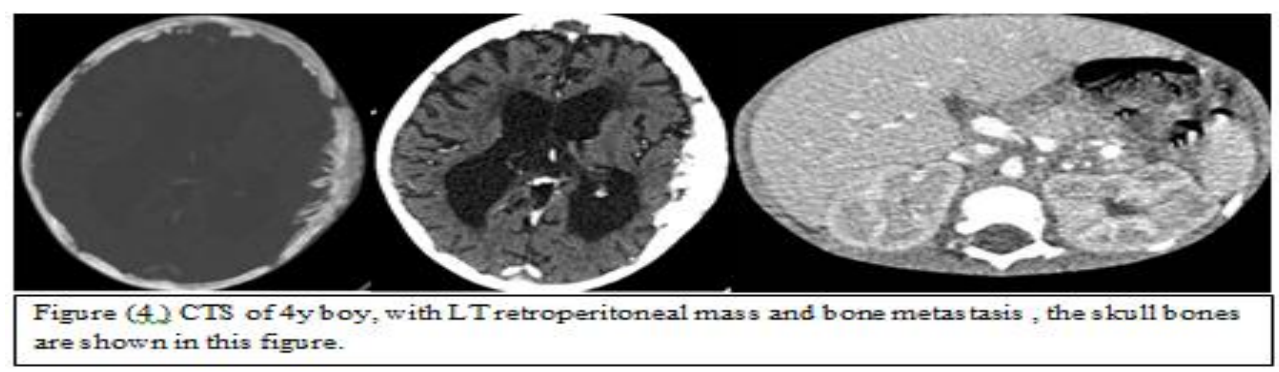




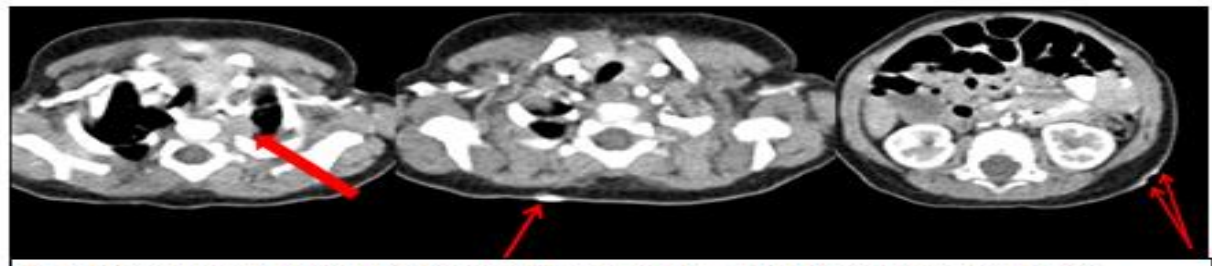

Figure (5) 8 months girl with LT superior medias tinal mass (bold arrow) and mu1tiple cutaneous lesions (thin arrows)
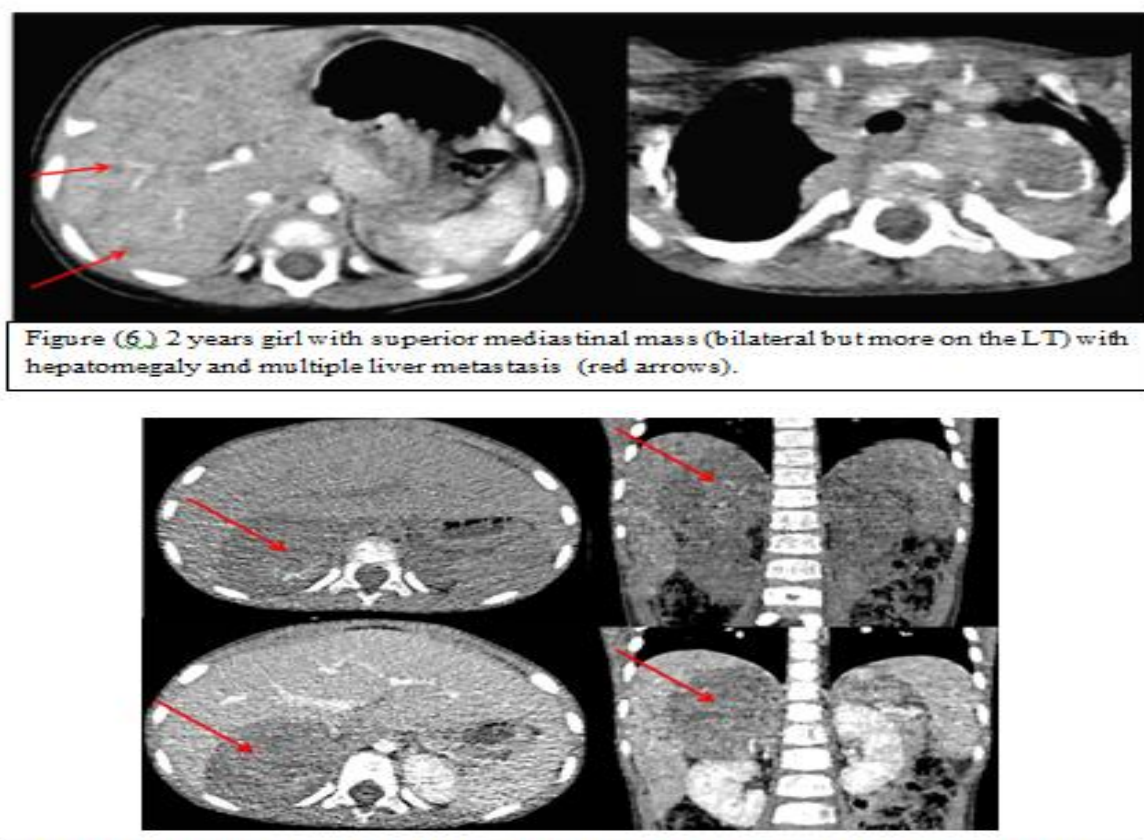

Figure (Z) 3 years gir1 with RT supra renal calcified heterogeneouslyenhancing mass (red arrow).

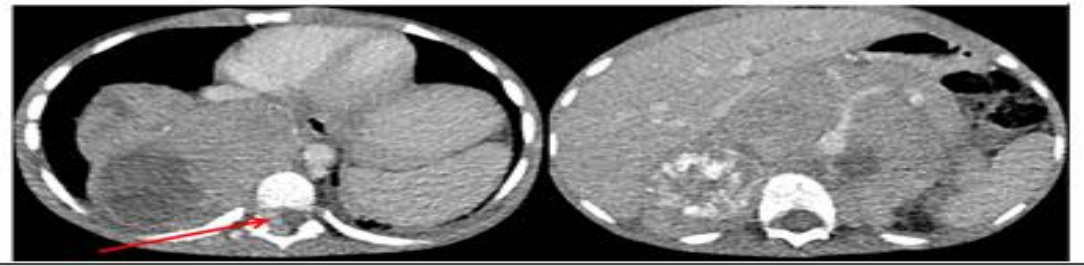

Figure (S) 7 years boy with extensive bilateral retroperitoneal, para-spinal abdominal and thoracic masses with spinal extension(red arrow). 


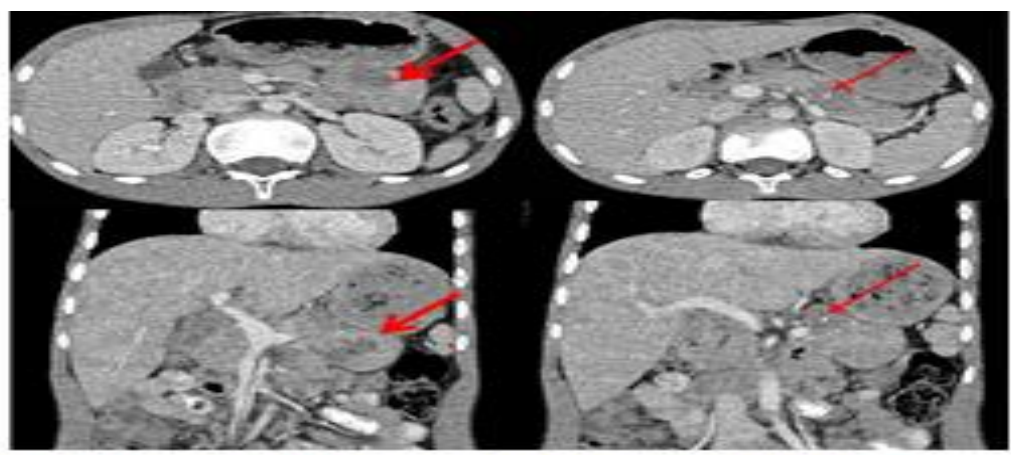

Figure (Q) 8 years boy IT retroperitoneal(organ of Zuackertbandi)

heterogeneously enhancing tissue soft mass (bold arrow), invading peroximal

aspect of pancreatic tail(thin arrow).

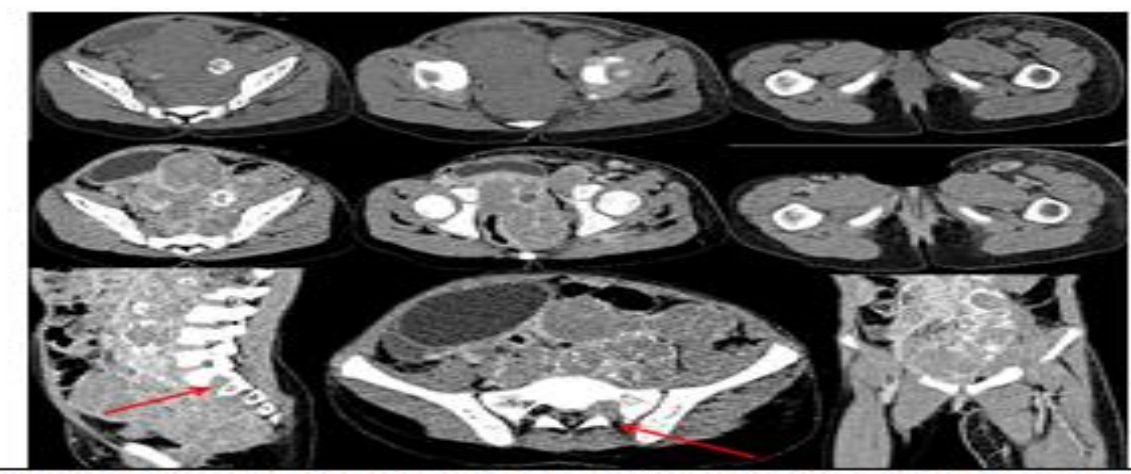

Figure $(10) 6$ years girl peresented with L T lower 1 imb swelling. There are multiple bilateral abdominopelwic retroperitoneal heterogeneousiy enhancing calcified soft tissue masses comperessing mainty the I T iliac vessels (vein) but parent vein, no thrombus, causing a swollen LT 1 ower $1 \mathrm{imb}$, with $L T$ sacral

\section{Discussion:-}

Effective patient's planning therapy requires precise delineation of the local extent of the neuroblastoma and evaluation of distant metastases. CT, MRI and bone scintigraphy are the primary imaging modalities used in staging disease in children with neuroblastoma. The imaging protocol might vary from institution to institution ${ }^{(9)}$.

CT was considered the imaging mode of choice for staging neuroblastoma in all sites ${ }^{(10)}$. Recently, MRI has supplemented CT for the staging of neuroblastoma ${ }^{(9,11,12,13)}$, and it is better for para-spinal lesions and is essential when assessing intra-foraminal extension of the tumor and its potential for cord compression ${ }^{(2)}$, but as there is some limitation in imaging modalities in our hospital (unavailability of MR scanner and scintigraphy), as many district hospitals that may not have the facilities for pediatric MRI, particularly in the age group prevalent for neuroblastoma, in whom general anesthesia is often required. Contrast-enhanced CT is therefore the most commonly used modality for disease staging for neuroblastoma worldwide ${ }^{(9)}$. Thus this study was conducted to evaluate the role of MDCT in diagnosis, extent and radiological characterization of neuroblastoma.

The total accuracy of MDCT in the current study in diagnosis of neuroblastoma and assessing its extent was $(83.3 \%)$, comparable with reported percentage ${ }^{(9)}$ and the total sensitivity was $(90.9 \%)$. The patients ages ranged from (5 months - to 8 years) with peak incidence was at $\left(2-3\right.$ years) comparable with other literatures ${ }^{(14,15,16)}$. The current study showed females predominance $(68.2 \%)$ while other literature showed that NB most often affects males $^{(14)}$.

Most common location was in the abdomen (77.3\% ), more than two third at the adrenal gland, where the least common sites were the neck and pelvis and the percentage was $(4.5 \%)$ for each, relatively comparable with other studies ${ }^{(1,14,17)}$. 
Local disease was assessed by CT, along with local extension on the same side in, but some limitation was found for assessment of invasive growth, for the paucity of fat surrounding the abdominal organs in small children, thus stage 1 was not reported and not stated in this current study, while only two were with stage 2 for local invasion. Lymph node involvement, INSS( International Neuroblastoma Staging System) stage 2B/3, cannot be assessed without surgical or percutaneous biopsy ${ }^{(1,5)}$. The current study showed that most of the tumors extend beyond the midline, diagrammatic extension and the presence of distant metastasis 18 out of 22 of patients had tumors of stages 3 or 4 and thus lymphadenopathy would not really affect the staging. Distant metastasis were found in more than half of the patients (59.1\%), almost all of them showed metastasis at time of presentation comparable with other literatures ${ }^{(5)}$, mostly to bone ( which is shown clearly on CTS bone algorithm) . Lung metastasis is rare ${ }^{(1)}$, CT is not surpassed by any of other imaging modalities for detection of lung metastasis ${ }^{(5)}$. Again calcification is better seen on CTS ${ }^{(5,10)}$, and it was seen in about $(72.7 \%)$ of the patients, comparable with other literatures $^{(5)}$ and it is a finding favors NB and according to some reports indicating an improved prognosis ${ }^{(5,18)}$. Stage $4 \mathrm{~S}$ was reported in $2(9.09 \%$ ) patients, that shows favorable prognosis with high survival rates and even spontaneous regression ${ }^{(14)}$.

\section{Conclusion:-}

MDCT is a valuable tool in diagnosis and radiological characterization of neuroblastoma.

\section{References:-}

1. Georgia Papaioannou and Kieran McHugh; Neuroblastoma in childhood: Review and radiological findings. 2005 International Imaging Society, 5:116-127

2. Maris JM, Hogarty MD, Bagatell R, Cohn SL. Neuroblastoma.Lancet 2007;369:2106-20.

3. Lonergan GJ, Schwab CM, Suarez ES et-al.(2002) Neuroblastoma, ganglioneuroblastoma, and ganglioneuroma: radiologic-pathologic correlation. Radiographics. 22 (4): 911-34.

4. De Bernardi B, Nicolas B, Boni L, Indolfi P, et al. Disseminated neuroblastoma in children older than one year at diagnosis: comparable resultswith three consecutive high-dose protocols adopted by the Italian Co-Operative Group for Neuroblastoma. J Clin Oncol.2003;21:1592-601.

5. C. Hugosson, R. Nyman, H. Jorulf, et al; (1999) Imaging of Abdominal Neuroblastoma in Children, Acta Radiologica, 40:5,534-542. Published online: 07 Jan 2010

6. BRODEUGR. M., PRITCHARJD., BERTHOLDE et al.: Revisions of the international criteria for neuroblastoma diagnosis, staging and response to treatment. J. Clin. Oncol.11 (1993), 1466.)

7. Kushner BH. Neuroblastoma: a disease requiring a multitude of imaging studies. J Nucl Med 2004; 45: 117288 .

8. Hiorns MP and Owens CM. Radiology of neuroblastoma in children. Eur Radiol 2001; 11: $2071-81$.

9. M CHU, 1D D RASALKAR, Y J HU etal; PICTORIAL REVIEW: Clinical presentations and imaging findings of neuroblastoma beyond abdominal mass and a review of imaging algorithm. The British Journal of Radiology, 84 (2011), 81-91.

10. David R, Lamki N, Fan S et-al. The many faces of neuroblastoma. Radiographics. 1989;9 (5): 859-82.

11. Ng YY, Kingston JE. The role of radiology in the staging of neuroblastoma. Clin Radiol 1993;47:226-35.

12. Siegel MJ, Ishwaran H, Fletcher BD, et al. Staging of neuroblastoma at imaging: report of the radiology diagnostic oncology group. Radiology 2002; 223:168-75.

13. Pfluger T, Schmied C, Porn U, et al. Integrated imaging using MRI and 123I metaiodobenzylguanidine scintigraphy to improve sensitivity and specificity in the diagnosis of pediatric neuroblastoma. AJR Am J Roentgenol 2003;181:1115-24.

14. I.Sánchez Paniagua, M. V. Trujillo Ariza, M. Liñares Paz et al ; Neuroblastoma: Clinical and radiological features in common and atypical locations. European society of radiology.www.myESR.org. 2015. EPOS. Educational Exhibit.

15. McHugh K and Fairhurst J.(2014). Paediatric neoplasms. Recommendations for cross-sectional imaging in cancer management, Second edition. Faculty of Clinical Radiology.The Royal College of Radiologists.

16. Brisse HJ, McCarville MB, Granata $\mathrm{C}$ et al. Guidelines for Imaging and Staging of Neuroblastic Tumors: Consensus Report from the International Neuroblastoma Risk Group Project. Radiology 2011; 261(1):243-257.

17. Ruppert David, Neela Lamki, Susan Fan, et al; The many faces of neuroblastoma. RadioGraphics1989,[9]: 859-881.

18. BRODEUGR. M. \& CASTLEBERRY. R.P: Neuroblastoma. In:Principles and practice of pediatric oncology, ch. 31. Editedby P. A. Pizzo \& D. G.Poplack. J. B. Lippincott Co., Philadelphia1993. 